\title{
Effects of cold aberrations on the longitudinal error signals and its compensation
}

\author{
P. Giri, ${ }^{a, b}, *$ D. Bersanetti, ${ }^{c}$ M. Boldrini, ${ }^{d, e}$ J. Casanueva Diaz, ${ }^{f}$ M. Cifaldi, ${ }^{g, h}$ V. \\ Fafone, ${ }^{g, h}$ M. Lorenzini, ${ }^{g, h}$ D. Lumaca, ${ }^{g, h}$ I. Nardecchia, ${ }^{h}$ M. Pinto, ${ }^{f}$ A. Rocchi, ${ }^{h}$ C. \\ Taranto $^{g, h}$ E. N. Tapia San Martín, ${ }^{i}$ M. Valentini, ${ }^{j, k}$ and M. Mantovani ${ }^{f}$ \\ ${ }^{a}$ Università di Pisa, I-56127 Pisa, Italy \\ ${ }^{b}$ Istituto Nazionale di Fisica Nucleare, Sezione di Pisa, I-56127 Pisa, Italy \\ ${ }^{c}$ Istituto Nazionale di Fisica Nucleare, Sezione di Genova, I-16146 Genova, Italy \\ ${ }^{d}$ Università di Roma "La Sapienza”, I-00185 Roma, Italy \\ e Istituto Nazionale di Fisica Nucleare, Sezione di Roma, I-00185 Roma, Italy \\ ${ }^{f}$ European Gravitational Observatory (EGO), I-56021 Cascina, Pisa, Italy \\ ${ }^{g}$ Università degli Studi di Roma Tor Vergata,I-00133,Rome, Italy \\ ${ }^{h}$ Istituto Nazionale di Fisica Nucleare, Sezione di Roma Tor Vergata, I-00133 Roma, Italy \\ ${ }^{i}$ Nikhef, Science Park 105, 1098 XG Amsterdam, Netherlands \\ ${ }^{j}$ Università di Trento, Dipartimento di Fisica, I-38123 Povo, Trento, Italy \\ ${ }^{k}$ Istituto Nazionale di Fisica Nucleare, Trento Institute for Fundamental Physics and Applications, I-38123 \\ Povo, Trento, Italy \\ E-mail: priyanka.giri@pi.infn.it
}

The target sensitivity of Advanced Virgo+ for the fourth observation run, O4 is about 90-120 $\mathrm{Mpc}$ in the BNS range. To achieve this, several hardware upgrades are under process. One of the most relevant upgrades concerns installation of the signal recycling mirror (SR), which forms an additional marginally stable cavity along with the power recycling cavity (PRC) which was already present in Advanced Virgo. The marginality of the recycling cavities makes longitudinal control very sensitive to aberrations. Thus, to compensate for the cold optical aberrations present in the DRMI of Advanced Virgo+, an upgrade of the system has been done to increase the power available for the central heating $(\mathrm{CH})$ actuator and make it compliant with the requirements for O4. The study is done for cold defects and their mitigation, in particular, their effects on the longitudinal error signals and on the sidebands' power, which are required for locking the DRMI of Advanced Virgo+. Thus, the focus of this paper is on Dual Recycled Michelson (DRMI) and the effects of cold aberrations on control of longitudinal degrees of freedom in Advanced Virgo+.

\footnotetext{
*** The European Physical Society Conference on High Energy Physics (EPS-HEP2021), ***

*** 26-30 July $2021 * * *$

*** Online conference, jointly organized by Universität Hamburg and the research center DESY ***
}

${ }^{*}$ Speaker 


\section{Introduction}

Ground-based gravitational wave detector, Advanced Virgo + (AdV+) [1] has been configured with an additional optic, a signal recycling mirror, SR, for improving the sensitivity in the high-frequency range during the fourth observation run, $\mathrm{O} 4$ [2]. For this optical configuration modification, a new locking acquisition strategy has been used, compared to what was implemented for $\mathrm{O}^{1}{ }^{1}$ (based on the one implemented in Advanced LIGO [3]). The main difference in the locking sequence used in Advanced Virgo+ and Advanced LIGO is due to the presence of two marginally stable cavities in DRMI in Advanced Virgo+, the signal recycling cavity (SRC) and the power recycling cavity (PRC) (with cavity stability parameter, g 0.9999885²), compared to stable cavities present in Advanced LIGO. These marginally stable conditions make the longitudinal control highly sensitive towards optical aberrations produced during the fabrication process, especially to the non-uniformity of the substrate transmission map, called 'cold' defects. This paper is focused on the effect of the cold defects on the longitudinal error signals.

\section{Lock acquisition strategy of DRMI}

To bring the interferometer from its uncontrolled condition to its final working point is called lock acquisition and requires control over five longitudinal degrees of freedoms (DOFs): DARM, CARM, MICH, PRCL, and SRCL (see Fig.1). DARM and CARM are the differential and common mode of the long arm cavities which we use to observe the GWs and stabilize the laser respectively. The required dark fringe of the interferometer is observed in MICH. PRCL and SRCL are the recycling cavities for power and signal respectively. And the control of these longitudinal DOFs is done by Pound-Drever-Hall (PDH) error signals [4]. However, the control of all five longitudinal degrees of freedom simultaneously is difficult. Thus, to reduce the longitudinal DOFs to be controlled at a time, the locking strategy is divided into several steps. The first step is to control DARM and CARM with an auxiliary laser while keeping arm cavities out of resonance from the main laser [5]. This makes DRMI locking with its three DOFs (MICH, PRCL, and SRCL) easier. Finally, the arm cavities are slowly brought to resonance along with locked DRMI to lock the full interferometer [6].

Hence, the first objective is to lock the DRMI in Advanced Virgo+ using PDH error signals, where the working point of DRMI of Advanced Virgo+ is achieved as follows: i) MICH in dark fringe ii) PRCL in resonance for 6 and $56 \mathrm{MHz}$ sidebands iii) SRCL in resonance for $56 \mathrm{MHz}$ sideband iv) Carrier anti-resonant in DRMI [7]. However, due to the presence of the cold defects in DRMI, a cavity mode-mismatch can be observed, in other words, the input laser modes do not match with the modes in the PRC and the SRC and the coupling coefficient between the laser TEM 00 and the recycling cavities TEM $_{00}$ becomes less than one [8]. In the presence of this effect, a decrease in the recycling cavity gain undermines the sidebands' power. And, since sidebands are required for locking the interferometer, it affects the sensitivity of the interferometer as an ultimate consequence.

\footnotetext{
${ }^{1}$ In O3, the central interferometer consisted of only PRC and Michelson (MICH) longitudinal DOFs

${ }^{2}$ A stable cavity should have $0<g<1$ however, in DRMI configuration of Advanced Virgo+, g 1, hence marginally stable. The reason for the cavity "unstability" is that the radius of curvature (RoC) of PR (1477 m) has been off by $47 \mathrm{~m}$ from its nominal configuration (1430 m), and now SR too.
} 


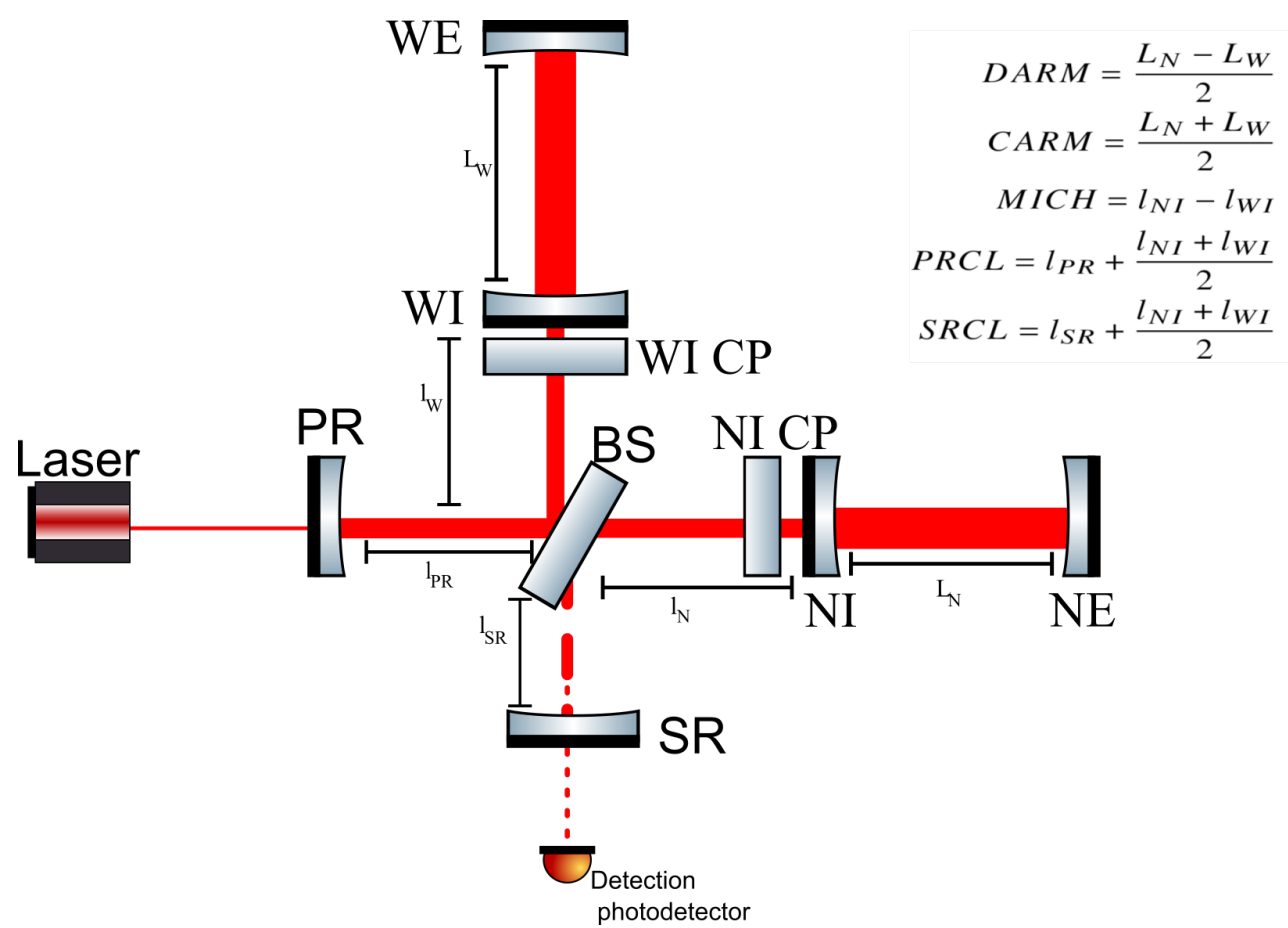

Figure 1: Optical layout of Advanced Virgo+ for $\mathrm{O}_{4}$ run. The schematic diagram shows the five longitudinal degrees of freedom.

The aftermath of cold defects is that the cavities are prone to generation of higher-order modes (HOMs) which are then recycled in the cavities due to low stability of the cavity. This has a direct consequence on the error signals, where an offset and reduction of optical gain in the longitudinal DOFs' error signals is observed [10]. This also reduces the linear region of the error signals (larger the linear region, easier to engage the lock) and low power of the sidebands which makes it difficult to validate the optical quality of the DRMI. Thus, compensation of cold defects is fundamental for bringing the interferometer to its optimal working point.

\section{Compensation of cold defects}

For compensating the thermal effects ${ }^{3}$ present in the Fabry-Perot cavities, a Double axicon system (DAS) has been used since $\mathrm{O} 3$ where an aberration complimentary to the one due to thermal effects is shined on the compensation plates ${ }^{4}$ (CPs) in both the arms [11]. This same compensation plate can also be used to mitigate the cold defects that lead to a change in the RoC of the PR, SR, and Compensation plates (CPs) from the ideal one. For example, other optics have hundreds of $\mathrm{km}$ of RoC except NI CP, which has $70 \mathrm{~km}$ of RoC and forms a converging lens. These cold defects affects the beam parameters and pointing, leading to different beam parameters for the sidebands and the carrier beam. To compensate both, for the cold defects and to provide a lens while the interferometer is unlocked in order to avoid thermal transients, an upgrade of the system devoted

\footnotetext{
${ }^{3}$ Note that the thermal effects are different from cold defects and are present due to power absorption in the arm cavities, resulting in thermo-elastic deformation in the optics.

${ }^{4} \mathrm{a}$ trasmissive optic placed close to each input test mass
} 


\begin{tabular}{c|ccc}
$\mathrm{DOF}^{\mathrm{OG}}$ & Ideal & with cold defects & after CH actuation \\
\hline$P R C L$ & $9.38 e+7$ & $5.30 e+7$ & $9.18 e+7$ \\
$M I C H$ & $2.93 e+6$ & $9.17 e+5$ & $2.51 e+6$ \\
SRCL & $1.40 e+6$ & $1.02 e+6$ & $1.39 e+6$
\end{tabular}

Table 1: Simulated optical gain (OG) of the three longitudinal DOFs in DRMI with cold defects and with $\mathrm{CH}$ on.

to the mitigation of optical aberrations has been done by implementing an improved version of the central heating ( $\mathrm{CH}$ ) benches 5 in Advanced Virgo+ [9].

Similar to how DAS compensates thermal effects by projecting an aberrations complementary to the one due to those effects, $\mathrm{CH}$ too mitigates cold defects by shining a beam on the $\mathrm{CP}$ which induces an optical aberration complimentary to that due to the cold defects. This strategy works for compensating the cold defects in the DRMI as the thermal lens induced by the $\mathrm{CH}$ in the $\mathrm{CP}$ substrate combines with the PR RoC as in Eqn.1, providing a correction of the cold defect. This brings DRMI to an almost stable configuration [12]

$$
\frac{1}{R o C_{P R}^{h o t}}=\frac{1}{R o C_{P R}^{c o l d}}+\frac{1}{f_{C P}^{h o t}} \text {, where } f \text { is the focal length of the hot CP. }
$$

The effectiveness of the $\mathrm{CH}$ actuation on the longitudinal DOFs error signals is being studied using the frequency domain modal simulation tool, FINESSE [13] and the results are shown in Fig.2. To have reliable results for marginally stable cavities from this modal simulation, a high number of HOMs have to be taken into account. The simulation was done for DRMI of Advanced Virgo+ while keeping RoC same for PR and SR (1430 m for each) with the only difference being \pm sign which was required according to the sign convention. In this condition, an ideal error signal (without any defect) was obtained for MICH, PRCL, and SRCL. Since the study is focused only on DRMI, arm cavities were kept out of resonance and DRMI was in a free-swinging mode.

For obtaining the effect of cold defects on the DRMI longitudinal error signals, a residual curvature was applied on NI CP and WI CP by changing the focal length of the CPs. Similarly, to obtain the effect of $\mathrm{CH}$ actuation on the error signals of the DRMI, a residual curvature which was the summation of the curvature induced by the $\mathrm{CH}$ and the cold lens ${ }^{6}$ of the $\mathrm{CPs}$, was applied. As expected, in presence of cold defects, the shape and the linear regions of the error signals for all three DOFs degraded significantly. On the other hand, while applying the residual curvature on the $\mathrm{CPs}$, after the actual $\mathrm{CH}$ actuation, a reliable error signal with higher optical gain (see Table1) and zero-crossing were obtained. ${ }^{7}$

The simulation's predictions on the effects of $\mathrm{CH}$ were validated with the experiment when $\mathrm{CH}$ was turned on in the interferometer. The residual curvature (or the $\mathrm{CH}$ power) considered in the simulations and the experiment were kept the same to compare the results. In Fig.3, the data considered was in the free-swinging configuration of DRMI for about 10 minutes in the two

\footnotetext{
${ }^{5} \mathrm{~A} \mathrm{CO}_{2}$ laser is chosen for this since its wavelength is completely absorbed by the fused silica optics (see Fig.1)

${ }^{6}$ Since they are complimentary, it was a subtraction of the two

${ }^{7}$ The $\mathrm{CH}$ residual curvature applied for the simulation study was the nominal one.
} 

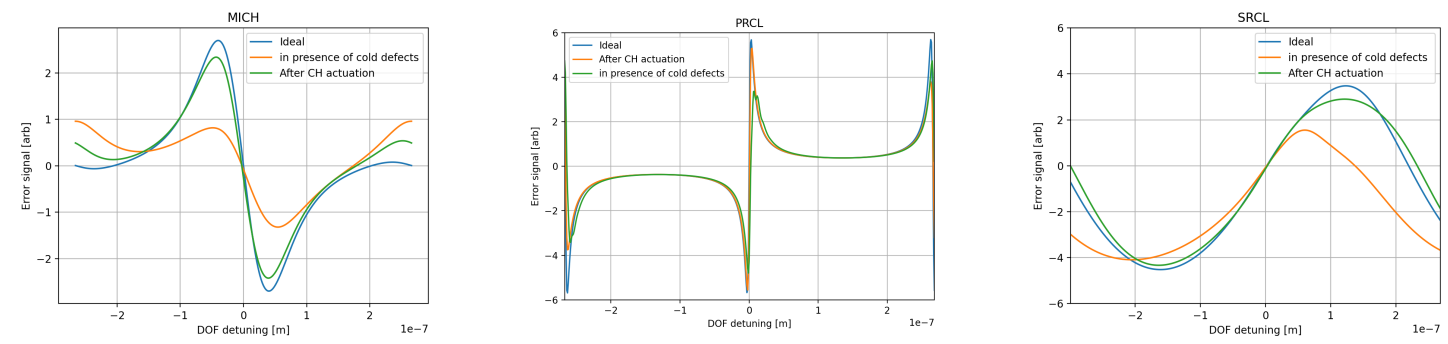

Figure 2: Error signals simulations for an ideal DRMI without any cold defects, DRMI in the presence of cold defects, and DMRI with central heating compensation ( $\mathrm{CH}$ on) for the three different DOFs. In the left-most plot, we show the MICH PDH error signal in the ideal case (blue curve), and the reduction of the optical gain in the presence of cold defects (orange curve). When $\mathrm{CH}$ actuation is applied, the recovery of the error signal zero-crossing and the optical gain (green curve) is visible. The middle and right-most show similar results for PRCL and SRCL respectively.

configurations i) DRMI in the presence of cold defects (TCS off) ii) DRMI when CH was turned on, in the interferometer (TCS on). For each data set, a region where DRMI working point condition was fulfilled ( $\mathrm{MICH}$ in dark fringe, 6 and 56MHz sidebands resonating and carrier anti-resonant), was selected. Then, for each point, the optical gain and zero-crossing were compared in the two configurations as specified above. It can be concluded from the data that after the $\mathrm{CH}$ actuation, an increase in the optical gain and reduction of the offset in the error signal has been achieved. Please note that only MICH and PRCL were considered in the measurement[14].
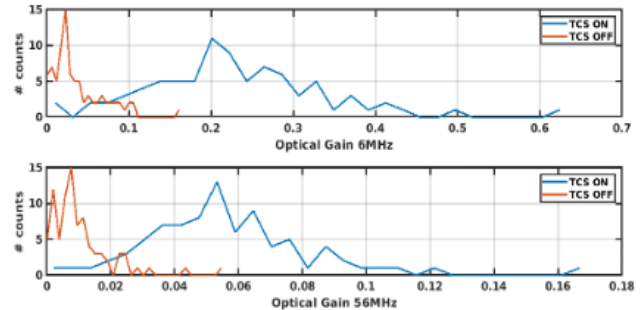
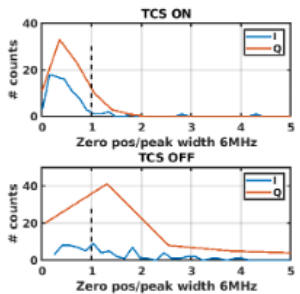
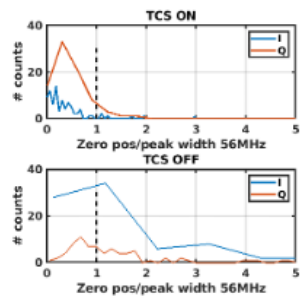

Figure 3: The figures on the left shows optical gain for PRCL (6MHz In-phase I) and MICH (56MHz In-phase I) when the CH was off (orange curve-TCS off) and after turning $\mathrm{CH}$ on (TCS on). The data was taken by keeping DRMI in free-swinging configuration for about 10 minutes. In right plots, the effect of Central heating on the zero-crossing of PRCL 6MHz (In-phase I) and MICH 56MHz(In-phase I). In the left plots an increase of optical gain in PRCL and MICH due to central heating is evident. And, on the right-hand side, the improvement in the offset of the zero-crossing of the PRCL and MICH error signals can be observed as when the $\mathrm{CH}$ actuation is on, the zero crossing is inside the peak of the sidebands while in the presence of cold defects only (TCS off) the offset on the error signals (most probably due to Homs) is so large that the zero crossing is outside the peak region.

The DRMI locking was achieved after the recovery of the error signals in the DRMI longitudinal DOFs. Further optimization of the optical gains and maximizing the sidebands power in the DRMI is being studied and some preliminary results to improve the optical gains in DRMI longitudinal DOFs are given in Fig.4. 

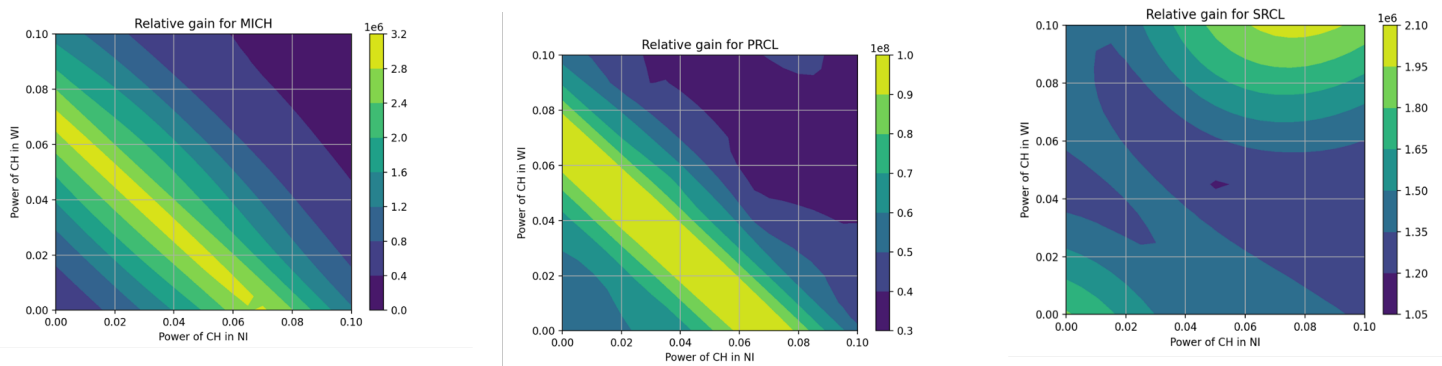

Figure 4: Relative optical gain for the three longitudinal degrees of freedom of DRMI. From left to right are MICH, PRCL, and SRCL respectively with different powers of $\mathrm{CH}$ actuation on the NI and WI CPs. These results were obtained considering only two HOMs in FINESSE.

\section{Conclusions and future work}

A stable lock of the two marginally stable cavities was achieved in Advanced Virgo+ with the assistance of $\mathrm{CH}$. $\mathrm{CH}$ actuation shows overall improvement in $\mathrm{OG}$ and offset reduction of the DRMI logitudnal DOFs error signals which is confirmed in simulations and from measurement. The quantitative difference in the OG and zero-crossing from the simulation and measurement could be due to an underestimation of the DRMI deviations in the simulations (see [15]). The effect of alignment has been previously studied which resulted a factor of 2 change in the OG [15]. Further investigation is currently going on to match the error measurements and simulations for the OG and offset in the longitudinal DOFs error signals.

\section{References}

[1] Acernese, F et al. (2015) Class. Quantum Grav. 32024001

[2] Abbott, B.P. et al. (2020) Living Rev Relativ 23, 3

[3] Vajente, G. (2014) ICRR Tokyo [DCC-G1400579-v1]

[4] Black, Eric D. (2000) American Journal of Physics 69, 79

[5] Rossi, C. et al. (2020) Galaxies 2020, 8(4), 87

[6] Martynov, D. (2015) CALTECH Dissertation (Ph.D.)

[7] Casanueva, J. and et al. (2012) Virgo Internal note [VIR-0327A-19]

[8] Rocchi, A. et al. (2012) J. Phys.: Conf. Ser. 363012016

[9] Giri, P. (2021) EPS-HEP

[10] Utina, A. (2020) Virgo internal note [VIR-0589A-20]

[11] Nardechhia, I. et al. (2021) Virgo Internal note [VIR-0536A-21]

[12] Cesarini, E. et al. (2017) Virgo Internal note [VIR-0266B-16]

[13] A Freise, A. et al. (2004) Class. Quantum Grav. 21 S1067

[14] Mantovani, M. (2021) Logbook entry-51431

[15] Valentini, M. (2019) Virgo Internal note [VIR-1031A-19] 